\title{
Equilibria and Stability in Partially Relaxed Plasma-Vacuum Systems
}

\author{
Hole M. J. ${ }^{1}$, Hudson S. R. ${ }^{2}$, and Dewar R. L. ${ }^{1}$
}

(1) Australian National University, ACT 0200, Australia (matthew.hole@anu.edu.au), (2) Princeton Plasma Physics Laboratories, P.O. Box 451, Princeton, New Jersey 08543, U.S.A.

\begin{abstract}
We develop a multiple interface variational model, comprising multiple Taylor-relaxed plasma regions, each of which are separated by an ideal MHD barrier. A principle motivation is the development of a mathematically rigorous ideal MHD model to describe intrinsically 3D equilibria, with nonzero internal pressure. A second application is the description of transport barriers as constrained minimum energy states. As a first example, we calculate the plasma solution in a periodic cylinder, generalizing the analysis of the treatment of Kaiser and Uecker, Q. Jl. Mech. Appl. Math.,57(1), 2004, who treated the single interface in cylindrical geometry. Expressions for the equilibrium field are generated, and equilibrium states computed. Unlike other Taylor relaxed equilibria, for the equilibria investigated here, only the plasma core necessarily has reverse magnetic shear. We show the existence of tokamak like equilibria, with increasing safety factor and stepped-pressure profiles. A stability treatment of the multiple barrier configuration reduces to an eigenvalue problem, where the eigenvectors are the normal displacements of the ideal barriers, and the eigen-matrix has tridiagonal structure. Next, marginal stability thresholds are explored in parameter space. For a single interface, results are benchmarked to Kaiser and Uecker. For multiple interfaces, we check our working via convergence tests, which reveal that the system approaches the single barrier case in the limit of vanishing interface width. The analysis provides a foundation upon which to study the stability of systems with a single internal barrier, placed at the reverse shear point.
\end{abstract}

\section{Introduction}

In 1967, Grad showed that in order for a static 3D equilibrium to exist, the pressure gradient $(\nabla p)$ must be zero in the neighbourhood of every rational flux surface, and poloidal flux $\psi_{p}$ surfaces must be relinquished. Since this time, this existence problem in 3D geometry has remained controversial. Our working develops a mathematically rigorous model of 3D ideal MHD confi gurations, in which the pressure is stepped, and $\nabla p$ is zero locally in any fi nite volume.

The original formulation of the 3D equilibrium problem, as proposed by Grad, was the search for a helical fi eld in cylindrical coordinates. Grad reduced the problem to the magnetic differential equation $\mathbf{B} \cdot \nabla \zeta=1$, with $\zeta$ a current potential. Next, Grad proved that unless stringent conditions are imposed on $\mathbf{B}$, the potential $\zeta$ is a non-integrable function due to resonances at rational rotational transform $\boldsymbol{l}$. If $\zeta$ is non-integrable, then $\mathbf{J}$ and the total current are infi nite, and the solution unphysical. A more transparent illustration, which elucidates the requirements on $\mathbf{B}$ and $\nabla p$ for arbitrary 3D confi gurations involves adding a helical fi eld perturbation $\delta \mathbf{B}$ to a 2D fi eld $\mathbf{B}_{0}$ with perfect flux surfaces [1]. The fi eld must always satisfy $\mathbf{J} \times \mathbf{B}=\nabla p$, hence $\mathbf{B}_{0} \cdot \nabla p_{0}=\left(\mathbf{B}_{0}+\delta \mathbf{B}\right) \cdot \nabla\left(p_{0}+\delta p\right)=0$. Here, $\mathbf{J}$ is the current density and $p_{0}$ and $\delta p$ the unperturbed and perturbed pressures. To fi rst order, we require

$$
\delta \mathbf{B} \cdot \nabla p_{0}=-\mathbf{B}_{0} \cdot \nabla \delta p .
$$

By employing magnetic coordinates $\left(\psi_{t}, \theta_{m}, \phi\right)$ for $\mathbf{B}_{0}$, with $\theta_{m}$ and $\phi$ magnetic poloidal and toroidal angles, and linearizing the perturbations

$$
\begin{aligned}
\delta p & =\sum_{m} \sum_{n} p_{m n} e^{i(n \phi-m \theta)}, \\
\frac{\delta \mathbf{B} \cdot \psi_{t}}{\mathbf{B}_{0} \cdot \nabla \phi} & =\sum_{m} \sum_{n} b_{m n} e^{i(n \phi-m \theta)}
\end{aligned}
$$


it can be shown that Eq. (1) reduces to

$$
i\left(n-m \imath\left(\psi_{t}\right)\right) p_{m n}=p_{0}^{\prime}\left(\psi_{t}\right) b_{m n} .
$$

Here, $n$ and $m$ are toroidal and poloidal mode numbers, and $p_{m n}$ and $b_{m n}$ perturbed pressure and magnetic fi eld Fourier coeffi cients. In general, and for a given $(m, n)$ perturbation within the range $\boldsymbol{l}_{\min }<n / m<\boldsymbol{l}_{\max }$ there will be a flux surface $\psi_{t}$ resonant with the perturbation (i.e. $\left.\imath\left(\psi_{t}\right)=n / m\right)$. For these cases, a nonzero 3D perturbation can exist only if $p_{0}^{\prime}\left(\psi_{t}\right)=0$. The existence of $3 \mathrm{D}$ equilibria can hence only be guaranteed providing $\nabla p_{0}=0$.

Fields for which $\nabla p=0$ are Beltrami fi elds, or force-free magnetic fi elds in astrophysical literature, where they were introduced over 50 years ago by Lüst and Schlüter [2] and Chandrasekhar [3], amongst others. The motivation for the work was the vanishing of the Lorenz $\mathbf{J} \times \mathbf{B}$ force, enabling astrophysical stationary state solutions. Woltjer [4] was the fi rst to derive a Beltrami fi eld by trying to minimize the total energy of a pressure-less plasma subject constant helicity. Whilst successfully describing the nature of stable solutions, Woltjer's working did not address how the plasma evolved to the lower energy state. Nearly 20 years later, Taylor [5-6] addressed this diffi culty by two conjectures initially developed to describe turbulent relaxation in the reverse fi eld pinch : magnetic helicity would be roughly conserved during the relaxation process, even in the presence of resistivity, and that no other topological invariant would survive the relaxation phase. Since these formulative works, a large body of literature has been devoted to force-free fi elds and Taylor relaxation. In astrophysical plasmas, important applications include coronal loops and accretion disks. In laboratory plasmas, examples include reverse fi eld pinches and spheromaks. The geophysical monograph "Magnetic Helicity in Space and Laboratory Plasmas" [7] provides an an overview, and lists seminal references.

A parallel advance in magnetically confi ned fusion plasma physics has been the discovery of high confi nement regimes, where at suffi ciently high heating power, the plasma self-organises to produce internal transport barriers. Whilst descriptive theories for these transport barriers exist (e.g. shear flow suppression of turbulence [8] and chaotic magnetic fi eld line dynamics [9]), little research has been devoted to addressing why the plasma self-organises into this state. One possible explanation is that these are constrained minimum energy states, where the plasma within the barrier satisfi es ideal MHD, and the plasma between barriers is in a Taylor relaxed state [6].

Our working builds principally upon a variational model developed by Spies et al [10], which comprised a plasma/vacuum/conducting wall system. In Spies [10] the theory is applied to a plasma slab equilibrium, with boundary conditions designed to simulate a torus. Later analysis by Spies [11] extended the plasma model to include fi nite pressure. In 2005, Kaiser and Uecker [12] analysed the fi nite pressure model in cylindrical geometry. More recently, Hole et al [13] extended the single interface cylindrical treatment of Kaiser and Uecker to multiple interfaces, and demonstrated the existence of partially relaxed Taylor plasmas with tokamak-like magnetic shear profi les. In this work, we perform a stability analysis on the stepped pressure profi le plasmas in cylindrical geometry. Our working also complements work by Hudson et al [14], which developed a numerical algorithm for the calculation of Beltrami fi elds in weakly asymmetric plasmas.

This paper is arranged as follows : Sec. 2 presents the variational model for the stepped pressure profi le equilibria. Equations for equilibrium and perturbed fi elds are derived, and expressions for plasma stability determined for local and global displacements. Section 3 solves for the 
equilibrium fi eld in cylindrical geometry, and generates a mapping between different equilibrium constraint representations. Next, Sec. 4 solves for the perturbed fi eld in a cylindrical plasma, and reduces the stability to an eigenvalue equation. The eigenvalue problem is solved numerically for one and two barrier systems. Finally, Sec. 5 contains concluding remarks.

\section{Multiple Interface Plasma-Vacuum Model}

We generalise the analysis of Kaiser and Uecker [12] to an arbitrary number $N$ of Taylor relaxed states, each separated by an ideal MHD barrier. The system is enclosed by a vacuum, and encased in a perfectly conducting wall. For such a system, the energy functional can be written

$$
W=\sum_{i=1}^{N} U_{i}-\sum_{i=1}^{N} \mu_{i} H_{i} / 2-\sum_{i=1}^{N} v_{i} M_{i}
$$

where $\mu_{i}$ and $v_{i}$ are Lagrange multipliers, and

$$
\begin{aligned}
U_{i} & =\int_{\mathscr{R}_{i}} d \tau^{3}\left(\frac{P_{i}}{\gamma-1}+\frac{B_{i}^{2}}{2}\right), \\
M_{i} & =\int_{\mathscr{R}_{i}} d \tau^{3} P_{i}^{1 / \gamma} \\
H_{i} & =\int_{\mathscr{R}_{i}} d \tau^{3} \mathbf{A} \cdot \nabla \times \mathbf{A}-\oint_{C_{s i}} \mathbf{d l} \cdot \mathbf{A} \oint_{C_{l i}} \mathbf{d l} \cdot \mathbf{A} .
\end{aligned}
$$

The term $U_{i}$ is the potential energy, $M_{i}$ the plasma mass, and $H_{i}$ the magnetic helicty in each region $\mathscr{R}_{i}$. In Eqs. (6) - (8), $d \tau^{3}$ is a volume element, $\gamma$ the ratio of specific heats, and $P_{i}, B_{i}$ and $\mathbf{A}_{i}$ the equilibrium pressure, magnetic fi eld strength and vector potential respectively. The regions $\mathscr{R}_{i}$ comprise the $N$ plasma regions $\mathscr{R}_{i}=\mathscr{P}_{i}$ and the vacuum region $\mathscr{R}_{N+1}=\mathscr{V}$. Each plasma region $\mathscr{P}_{i}$ is bounded by the outer and inner ideal MHD interfaces $\mathscr{I}_{i}$, and $\mathscr{I}_{i-1}$ respectively, whilst the vacuum is encased by the perfectly conducting wall $\mathscr{W}$. Finally, $C_{s i}$ and $C_{l i}$ are fi xed loops the short and long way around $\mathscr{R}_{i}$. Figure 1(a) shows the geometry of the system.

Setting the fi rst variation to zero yields the following set of equations:

$$
\begin{array}{ccl}
\mathscr{P}_{i} ; & \nabla \times \mathbf{B}=\mu_{i} \mathbf{B}, & P_{i}=\text { const. } \\
\mathscr{I}_{i} ; & \mathbf{n} \cdot \mathbf{B}=0, & \left\langle P_{i}+1 / 2 B^{2}\right\rangle=0 \\
\mathscr{V} ; & \nabla \times \mathbf{B}=0, & \nabla \cdot \mathbf{B}=0 \\
\mathscr{W} ; & \mathbf{n} \cdot \mathbf{B}=0 &
\end{array}
$$

where $\mathbf{n}$ a unit vector normal to the plasma interface $\mathscr{I}_{i}$, and $\langle x\rangle=x_{i+1}-x_{i}$ denotes the change in quantity $x$ across the interface $\mathscr{I}_{i}$. The boundary conditions on $\mathbf{n} \cdot \mathbf{B}$ arise because each interface and the conducting wall is assumed to have infi nite conductivity. In turn, these imply the following flux constraints during Taylor relaxation:

$$
\begin{aligned}
\mathscr{P}_{i} & ; \quad \Psi_{P, i}^{t}=\mathrm{const}, \\
\mathscr{V} & ; \quad \Psi_{V}^{t}=\mathrm{const}, \quad \Psi_{V}^{p}=\mathrm{const},
\end{aligned}
$$

where the subscripts $P_{i}, V$ are labels for quantities within the i'th plasma region, and vacuum region respectively, and the superscripts $p, t$ label the fluxes as poloidal and toroidal, respectively. Given the vessel with boundary $\mathscr{W}$, the interfaces $\mathscr{I}_{i}$, and the magnetic fi eld B, Eqs. (9)-(12) constitute a boundary problem for the plasma pressure $P_{i}$ in each region $\mathscr{R}_{i}$. 

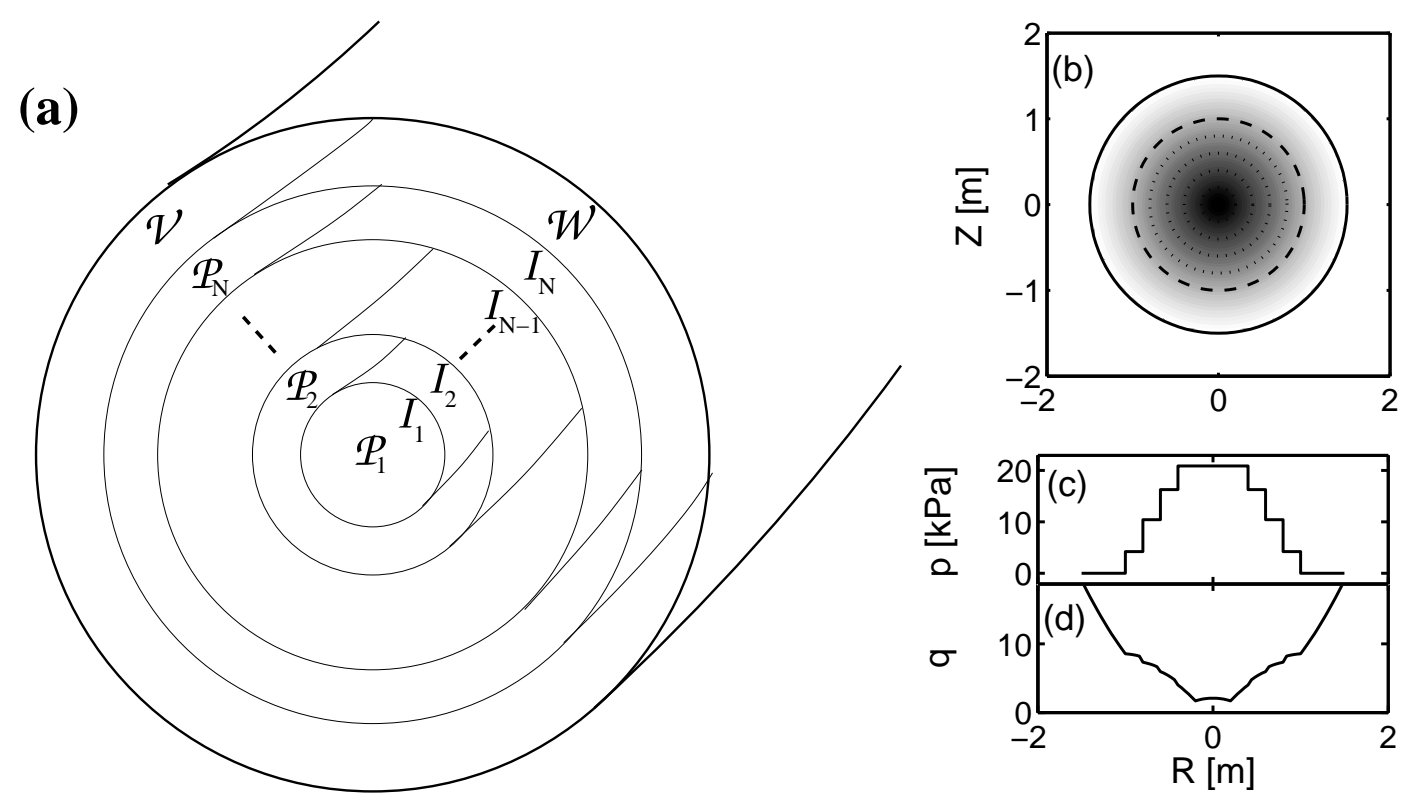

Figure 1: Schematic of magnetic geometry (a), showing ideal MHD barriers $\mathscr{I}_{i}$, the conducting wall $\mathscr{W}$, plasma regions $\mathscr{P}_{i}$ and the vacuum $\mathscr{V}$; and example stepped-pressure plasma profi le solution in cylindrical geometry, (b)-(d). Panel (b) is a shaded contour plot of the polodial flux, where the dashed line is the vacuum boundary, and the dotted lines the ideal barriers within the plasma. Panels (c) and (d) show the pressure profi le and safety factor profi le, respectively.

The second variation is a straightforward generalization of Spies $[10,11]$ to multiple interfaces. That is,

$$
\delta^{2} W=\sum_{i}^{n}\left(\delta^{2} W_{P, i}+\delta^{2} W_{I, i}\right)+\delta^{2} W_{V}
$$

where

$$
\begin{aligned}
\delta^{2} W_{P, i} & =\int_{\mathscr{P}_{i}} d \tau^{3}\left(|\nabla \times \mathbf{a}|^{2}-\mu_{i} \mathbf{a}^{*} \cdot \nabla \times \mathbf{a}+\left|p_{i}\right|^{2} / \gamma_{i} P_{i}\right) \\
\delta^{2} W_{I, i} & =\int_{\mathscr{I}_{i}} d \sigma^{2}\left|\xi_{i}\right|^{2}\langle B \mathbf{n} \cdot \nabla B\rangle \\
\delta^{2} W_{V} & =\int_{\mathscr{V}} d \tau^{3}|\nabla \times \mathbf{a}|^{2}
\end{aligned}
$$

and where, following Kaiser and Uecker[12], we have used upper case symbols to denote equilibrium quantities, and lower case use to represent perturbation. Hence, $\mathbf{a}$ is the perturbed vector potential and $p_{i}$ the perturbation in the equilibrium pressure $P_{i}$. The term $\gamma_{i}$ is the ideal gas constant in each region, whilst $\xi_{i}=\xi_{i} \cdot \mathbf{n}$ denotes the normal displacement of $I_{i}$.

In Spies [10] the condition $\delta^{2} W>0$ was re-formulated as a highly nonlinear eigenvalue problem. That is, the functional $\delta^{2} W$ has been minimized subject to the constraint of constant $N_{A}$, where

$$
N_{A}=\int_{\mathscr{P} \cup \mathscr{V}} d^{3} \tau|\nabla \times \mathbf{a}|^{2}
$$

To solve the problem the Lagrangian multiplier $\lambda$ was introduced, and the functional $L=$ $\delta^{2} W-\lambda N_{A}$ varied. Solutions of $\delta L=0$ with $L=0$ are stable providing $\lambda>0$. 
For $N \geq 1$, reduction to a linear eigenvalue problem is possible with a different choice of normalization:

$$
N_{B}=\sum_{i}^{N} \int_{\mathscr{I}_{i}} d^{2} \sigma\left|\xi_{i}\right|^{2}
$$

where we have recognized that the displacement of the wall is zero, $\xi_{N+1}=0$. For $\mathscr{P}_{i}, \mathscr{V}, \mathscr{W}$, solutions of $\delta L=0$ can be written in terms of the perturbed magnetic fi eld $\mathbf{b}=\nabla \times \mathbf{a}$ as follows:

$$
\begin{aligned}
\mathscr{P}_{i} ; & \nabla \times \mathbf{b}=\mu_{i} \mathbf{b}, \\
\mathscr{I}_{i} ; & \xi_{i}^{*}\langle\mathbf{B} \cdot \mathbf{b}\rangle+\xi_{i}^{*} \xi_{i}\langle B(\mathbf{n} \cdot \nabla) B\rangle-\lambda \xi_{i}^{*} \xi_{i}=0, \\
& \mathbf{n} \cdot \mathbf{b}_{i, i+1}=\mathbf{B}_{i, i+1} \cdot \nabla \xi_{i}+\xi_{i} \mathbf{n} \cdot \nabla \times\left(\mathbf{n} \times \mathbf{B}_{i, i+1}\right), \\
\mathscr{V} \quad ; & \nabla \times \mathbf{b}=0 \\
& \nabla \cdot \mathbf{b}=0 \\
\mathscr{W} \quad ; & \mathbf{n} \cdot \mathbf{b}=0 .
\end{aligned}
$$

Equations (23) and (26) are boundary conditions, and do not result from setting $\delta L=0$. Employing a suitable Fourier decomposition, Eq. (23) solves for the unknown coeffi cients of the perturbed fi eld in each region. With substitution, Eq. (22) then becomes a linear eigenvalue equation. The set of equations is completed by expressions for the perturbed fluxes through each region.

\section{Cylindrical Equilibria}

In this section cylindrically symmetric equilibrium solutions are generated. A cylindrical coordinate system is used $(r, \theta, z)$, with equilibrium variations permitted only in the radial direction. Following Kaiser we use the normalization of plasma-vacuum boundary $r=1$, and assume that the cylinder is periodic in the $z$ direction, with periodicity $L$. In this system, solutions to Eqs. (9) - (12) can be written in vector notation $\mathbf{B}=\left\{B_{r}(r), B_{\theta}(r), B_{z}(r)\right\}$ as

$$
\begin{array}{llccl}
\mathscr{P}_{1} & : \mathbf{B}=\{0, & k_{1} J_{1}\left(\mu_{1} r\right), & k_{1} J_{0}\left(\mu_{1} r\right) & \}, \\
\mathscr{P}_{i} & : \mathbf{B}=\{0, & k_{i} J_{1}\left(\mu_{i} r\right)+d_{i} Y_{1}\left(\mu_{i} r\right), & k_{i} J_{0}\left(\mu_{i} r\right)+d_{i} Y_{0}\left(\mu_{i} r\right) & \}, \\
\mathscr{V} & : \mathbf{B}=\{0, & B_{\theta}^{V} / r, & B_{z}^{V} & \},
\end{array}
$$

where $k_{i}, d_{i} \in \Re$, and $J_{0}, J_{1}$ and $Y_{0}, Y_{1}$ are Bessel functions of the fi rst kind of order 0 , 1 , and second kind of order 0,1 , respectively. The terms $B_{\theta}^{V}$ and $B_{z}^{V}$ are constants. The constant $d_{1}$ is zero in the plasma core $\mathscr{P}_{1}$, because the Bessel functions $Y_{0}\left(\mu_{1} r\right)$ and $Y_{1}\left(\mu_{1} r\right)$ have a simple pole at $r=0$ [15]. The geometry of this system is analogous to the general screw pinch [16], but with key differences. Notably, the pressure gradient is zero, except at ideal MHD barriers, where it is a delta function.

With an analytic form for the equilibrium magnetic fi eld available, the equilibrium problem can now be prescribed in parameter space. Recognising that the change in pressure can be expressed in terms of the change in fi eld strength $B$ of the barriers, we observe that the plasma equilibrium is completely determined by the magnetic fi eld profi le and the radial position of the barriers. That is, the equilibrium is constrained by the $4 N+1$ parameters:

$$
\left\{k_{1}, \ldots, k_{N}, d_{2}, \ldots, d_{N}, \mu_{1}, \ldots, \mu_{N}, r_{1}, \ldots r_{N-1}, r_{w}, B_{\theta}^{V}, B_{z}^{V}\right\}
$$

where $r_{i}$ are the radial positions of the $N$ ideal MHD barriers, and $r_{w}$ the radial position of the conducting wall. Equivalently, the equilibrium can be constrained by the safety factors and magnetic fluxes. That is, the $4 N+1$ quantities

$$
\left\{\Psi_{1}^{t}, \ldots, \Psi_{N}^{t}, \Psi_{1}^{p}, \ldots, \Psi_{N-1}^{p}, \Psi_{V}^{t}, \Psi_{V}^{p}, q_{1}^{i}, \ldots, q_{N}^{i}, q_{1}^{o}, \ldots, q_{N}^{o}\right\}
$$


where $q_{i}^{i}$ and $q_{i}^{o}$ are the safety factor on the inside and outside of each interface. In cylindrical geometry the safety factor expands as

$$
q_{i}^{i}=\frac{2 \pi r_{i}}{L} \frac{B_{z, i}\left(r_{i}\right)}{B_{\theta, i}\left(r_{i}\right)}, \quad q_{i}^{o}=\frac{2 \pi r_{i}}{L} \frac{B_{z, i+1}\left(r_{i}\right)}{B_{\theta, i+1}\left(r_{i}\right)},
$$

whilst the toroidal and poloidal fluxes compute as follows:

$$
\begin{gathered}
\Psi_{i}^{t}=\int_{r_{i-1}}^{r_{i}} B_{z}(r) r d \theta d r=\frac{2 \pi}{\mu_{i}}\left[k_{i} r J_{1}\left(r \mu_{i}\right)+d_{i} r Y_{1}\left(r \mu_{i}\right)\right]_{r_{i-1}}^{r_{i}}, \\
\Psi_{i}^{p}=\quad \int_{r_{i-1}}^{r_{i}} B_{\theta}(r) L d r=\frac{-L}{\mu_{i}}\left[k_{i} J_{0}\left(r \mu_{i}\right)+d_{i} Y_{0}\left(r \mu_{i}\right)\right]_{r_{i-1}}^{r_{i}} .
\end{gathered}
$$

In the vacuum region, the fluxes compute as

$$
\Psi_{V}^{t}=B_{z}^{V} \pi\left(r_{w}^{2}-1\right), \quad \Psi_{V}^{p}=B_{\theta}^{V} L \ln r_{w} .
$$

Figure 1(b)-(d) shows an example with 5 ideal barriers. In addition to the poloidal flux, the safety factor is plotted, which necessarily decreases in the plasma core. In the plasma core, $q=r J_{0}\left(\mu_{1} r\right) / J_{1}\left(\mu_{1} r\right)$, which for all $\mu_{1}>0$ is a strictly decreasing function with increasing radius. Elsewhere $q$ may increase or decrease, depending upon the values of $d_{i} / k_{i}$ and $\mu_{i}$. In general, $q$ can jump at the interfaces, although the example shown here is chosen with $\delta q=0$.

\section{Cylindrical plasma stability}

Fourier decomposition of the perturbed fi eld $\mathbf{b}=\nabla \times \mathbf{a}$ and the displacements $\xi_{\mathrm{j}}$ at each interface enables a straight forward solution. That is,

$$
\mathbf{b}=\widetilde{\mathbf{b}} e^{i(m \theta+\kappa z)}, \quad \xi_{i}=X_{i} e^{i(m \theta+\kappa z)},
$$

where $m, \kappa$ are the Fourier poloidal mode-number and axial wave-number, and $\widetilde{\mathbf{b}}$ and $X_{i}$ are complex Fourier amplitudes. Under these Fourier substitutions, and after solving for the fi eld in each plasma region, the system of Eqs. (21) - (26) is reduced to the eigenvalue equation,

$$
\eta \cdot \mathbf{X}=\lambda \mathbf{X}
$$

with $\eta$ is a $N \times N$ matrix. The $i$ 'th row of $\eta$ is the $i$ 'th interface calculation of

$$
\left(\langle\mathbf{B} \cdot \mathbf{b}\rangle+\xi_{i}\langle B(\mathbf{n} \cdot \nabla) B\rangle\right) e^{-i(m \theta+\kappa z)}
$$

which is the fi rst two terms of Eq. (22), divided by $X_{i}^{*}$. In Eq. (36) $\mathbf{b}$ and $\mathbf{B}$ take values either side of the interface. In regions $\mathscr{R}_{i}$ and $\mathscr{R}_{i+1}$, Eq. (23) solves for $\widetilde{b}_{r}$ in terms of equilibrium quantities and the complex amplitudes $X_{i}, X_{i-1}$ and $X_{i+1}, X_{i}$, respectively. As such, $\eta$ is a tridiagonal matrix.

We have solved Eq. (35) for the set of $N$ eigenvalues $\lambda_{1}, \ldots, \lambda_{N}$, and eigenvectors $\mathbf{X}_{1}, \ldots, \mathbf{X}_{N}$ using standard numerical packages. First, using Mathematica, Fortran 90 statements were generated to compute the coeffi cients of the matrix $\eta$ for all cases. For each matrix element $\eta_{i j}$, the statements were coded into a case-selection algorithm. To determine the eigenvalue, the QR algorithm for real Hessenberg matrices was employed [17].

When evaluated for an eigenfunction, $\delta L$ vanishes, and so $\delta^{2} W=\lambda N_{B}$. The system is stable providing there do not exist eigenfunctions with $\lambda<0$. For each $m$ and magnetic confi guration, we have computed the spectrum of eigenvalues, as a function of $\kappa$. Marginal stability thresholds 
were investigated by sweeping $\kappa$ over the range $-K \leq \kappa \leq K$, with $K=20$ and $\delta \kappa=0.002$, and detecting changes in sign of any of the eigenvalues $\lambda$.

For $N=1$, Eq. (35) reduces to an expression for the eigenvalue $\lambda$. We have benchmarked our variational approach to the results of Kaiser and Uecker [12], in which marginal stability scans are available. Figure 2 is a plot of the $m=1$ and $m=2$ marginal stability boundaries as a function in $\mu_{1}, \delta$ space, with $r_{l}=1.1$, and for a selection of pressure values. Kaiser and Uecker defi ne $\delta$ to be a measure of the increase in pitch angle of the fi eld at the such that

$$
\begin{aligned}
B_{\theta, V} & =J_{1}\left(\mu_{1}\right) \cos \delta+J_{0}\left(\mu_{1}\right) \sin \delta \\
B_{z, V} & =J_{0}\left(\mu_{1}\right) \cos \delta-J_{0}\left(\mu_{1}\right) \sin \delta
\end{aligned}
$$

For consistency with our working, we map $\delta$ to the jump in safety factor

$$
\Delta q=\frac{2 \pi}{L}\left(\frac{J_{0}\left(\mu_{1}\right) / J_{1}\left(\mu_{1}\right)-\tan \delta}{1+J_{0}\left(\mu_{1}\right) / J_{1}\left(\mu_{1}\right) \tan \delta}-1\right)
$$

Different internal pressures are described by $\beta$. We generalize the defi nition of Kaiser and Uecker to multiple interfaces,

$$
\beta=\frac{2|| P_{i}||}{\left.B^{2}\right|_{r=1+}}
$$

where $\|$ denotes volume averaging. The term $\beta$ is related to our working by $k_{1}=\sqrt{1-\beta}$. Comparison of Fig. 2 to Fig. 3 in Kaiser and Uecker [12] shows the stability boundaries to be identical.
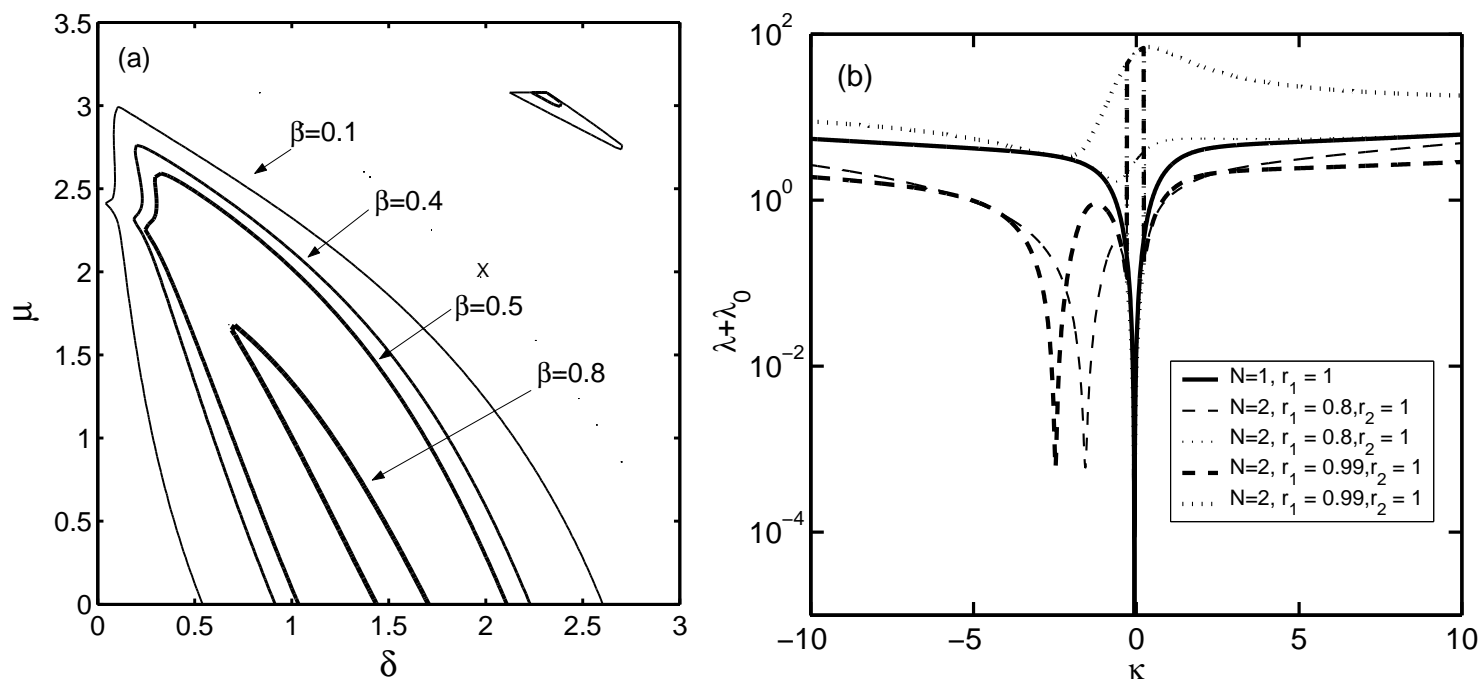

Figure 2: Figure (a) shows marginal stability boundaries for $m=1$ in $\mu_{1}-\delta$ space, and for different plasma $\beta$ values. The plasma has $r_{w}=1.1$ and $L=1$. The stable region is interior to each locus. The cross-hairs denote the equilibrium confi guration used for the dispersion curves presented in Fig. (b), which is a dispersion curve for $N=2$ and $m=1$, and for different internal barrier positions $r_{1}$.

To develop confi dence in the model, we next explore two interfaces $(N=2)$, in limiting regimes where the internal interface is expected to only weakly perturb the single interface eigenvalue. One such regime is for $m=1$, in the limit that radial position $r_{1}$ of the the inner interface $\mathscr{I}_{1}$ 
approaches the plasma-vacuum boundary $\mathscr{I}_{2}$ at $r_{2}=1$. Figure 2 shows a set of $m=1$ dispersion curves for varying separation $\varepsilon=r_{2}-r_{1}$ between the two interfaces. As the separation between the interfaces approaches zero, $\lambda_{1} \rightarrow \infty$, and $\lambda_{2} \rightarrow 2 \lambda(N=1)$. The eigenvalue $\lambda_{1}$ has eigenvector $\mathbf{X}_{1}=(1 / \sqrt{2},-1 / \sqrt{2})$, and corresponds to no free energy in the region $r_{1}<r<r_{2}$. The eigenvalue $\lambda_{2}$ has eigenvector $\mathbf{X}_{2}=(1 / \sqrt{2}, 1 / \sqrt{2})$, and corresponds to $N=1$ confi guration.

\section{Conclusions}

We have formulated a variational model for multiple interface stepped pressure profi le plasma confi gurations. The working extends previous treatments, which developed models for a single interface plasma-vacuum systems. The motivation for the work is the rigorous development of a model capable of generating 3D ideal MHD equilibria in arbitrary geometry. The system comprises multiple Taylor-relaxed plasma regions, each of which is separated by an ideal MHD barrier of zero width. The system is enclosed by a vacuum region, and encased by a perfectly conducting wall. As a fi rst step, analytic solutions were developed for the equilibrium and perturbed fi elds of a a multiple interface cylinder. For these equilibria, the safety factor in the core necessarily decreases monotonically. For regions outside of the innermost ideal barrier, solutions can be constructed with increasing safety factors, and decreasing pressures. A tokamak like example of a multiple-interface equilibria was provided.

System stability was examined by reducing expressions for the perturbed fi elds to an eigenvalue problem. For a single interface, marginal stability thresholds reduce to previous working. For multiple interfaces, system stability converges to the single interface case in the limit that the interface separation reduces to zero. The analysis provides a foundation upon which to study the stability of systems with a single internal barrier, placed at the reverse shear point. Initial results will be presented in tokamak like confi gurations as a function of $q_{\text {min }}$, and $q_{0}$.

This work was supported by Australian Research Council grant DP0452728.

\section{References}

[1] A. H. BOOZER, Rev. Mod. Physics, 76(4):1071-1141, 2004.

[2] R. LUST and A. SCHLUTER, Zs. f. Ap., 34(263), 1954.

[3] S. CHANDRASEKHAR, Proc. Nat. Acad. Sci., 42(1), 1956.

[4] L. WOLTJER, Proc. National Academy of Sciences, 44(6), 1958.

[5] J. B. TAYLOR, Phys. Rev. Lett., 33:1139, 1974.

[6] J. B. Talyor, Rev. Mod. Phys., 58(3):741-763, 1986.

[7] M. R. BROWN, R. C. CANFIELD, and A. A. PEVSTOV, Am. Geophysical Union, 1999.

[8] K. H. BURREL, Phys. Plas., 4(5):1499-1518, 1997.

[9] J. H. MISGUICH, Phys. Plas., 8(5):2132-2138, 2001.

[10] G. O. SPIES, D. LORTZ, and R. KAISER, Phys. Plas., 8(8):3652-3663, 2001.

[11] G. O. SPIES, Phys. Plas., 10(7):3030-3031, 2003.

[12] R. KASIER and H. UECKER, Q. Jl. Mech. Appl. Math., 57(1):1-17, 2004.

[13] M. J. HOLE, S. R. HUDSON, and R. L. DEWAR, Journal of Plasma Physics, 2006.

[14] S. R. HUDSON, M. J. HOLE, and R. L. DEWAR, Physics of Plasmas, 2006.

[15] M. ABROMOWITZ and I. A. STEGUN, Dover, 1972.

[16] J. P. FRIEDBERG, "Ideal Magnetohydrodynamics", Plenum Press, 1987.

[17] W. H. PRESS et al., Univ. of Cambridge Press, 2nd edition, 1997. 